\title{
A MICRO GAS PRECONCENTRATOR WITH IMPROVED PERFORMANCES FOR ENVIRONMENTAL MONITORING
}

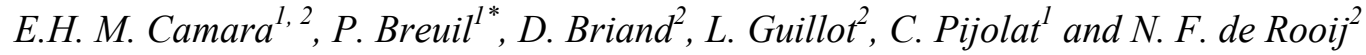 \\ ${ }^{1}$ Ecole Nationale Supérieure des Mines, Centre SPIN, LPMG-UMR CNRS 5148, Saint-Etienne, FRANCE \\ ${ }^{2}$ Ecole Polytechnique Fédérale de Lausanne, Institute of Microengineering, Neuchâtel, SWITZERLAND
}

\begin{abstract}
This paper presents the optimization of a micro gas preconcentrator applied to environmental monitoring. Different types of adsorbent materials introduced in silicon microchannels with various designs have been investigated and a special focus has been dedicated to the modes of operation of the preconcentrator. The optimization of the device and its operation were driven by its future application in outdoor environments. Parameters such as the preconcentration factor, cycle time and the influence of the humidity were considered along the optimization process. As a result of this study, a preconcentrator with a total cycle time of 10 minutes and the use of single wall carbon nanotubes (SWCNTs) as adsorbent exhibits a good preconcentration factor for VOCs with a limited influence of the humidity.
\end{abstract}

\section{KEYWORDS}

Pre-concentrator, Gas sensors, Micro-channel, Porous silicon, Carbon nanotubes,

\section{INTRODUCTION}

In a previous study [1, 2], a gas pre-concentrator based on porous silicon micro-channels filled with carbon nanopowders from a fluidic solution has been developed. The present work aims to improve the performances of this device, with new approaches in the design as well as in the choice of adsorbent materials and modes of operation. We have further investigated the contribution of porous silicon in the adsorption and desorption phases since besides the increase of its specific surface area as reported in [1], porous silicon when coupled with the adequate adsorbent can also modify the kinetics of desorption of the target gas.

Alternative absorbent materials such as carbon nanotubes and polymers, whose surface areas, pores-size distributions, and structures can be varied to serve as conformal adsorbents, were evaluated for the improvement of the performances of the preconcentrator [3].

The devices in this study have been developed targeting the preconcentration of benzene which is a representative gas for several types of applications relevant from atmospheric pollution control.

\section{EXPERIMENTAL}

\section{Preconcentrator design}

Micro-channels with three different shapes were designed in order to ease the introduction of the carbon nanopowders. The Figure 1 shows two micro-channels respectively in "Straight" and "Zigzag" designs with the magnification of its pillars.

The "Zigzag" design contributes to improve the carbon deposition by stopping it when injected into the microchannel. While the "Straight" design leads to an increase of the available area for deposition since the surface of the walls constitute a binding area for adsorbent particles. And lastly, the "Neutral" design without pillars was made in order to have a reference to determine the benefits or not to have pillars. At the inlet and outlet, a system of channels was designed targeting a uniform distribution of the gas or liquid flow. The walls of the channels can be made of silicon or porous silicon.
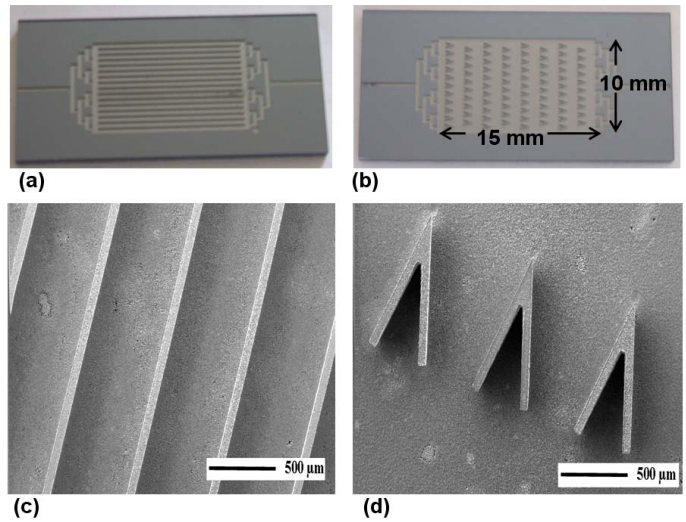

Figure 1: (a) Top view of a micro-channel in "Straight" design - (b) Top view of a micro-channel in "Zigzag" design - (c) Magnification of walls in straight design - (d) Magnification of pillars in "Zigzag" design.

\section{Preconcentrator fabrication}

The processing of these preconcentrators is simple. Following a photolithographic step with a thick AZ4562 photoresist, the inlet, outlet and reaction chamber of the micro-channel are DRIE etched in the silicon wafer to a depth of $325 \mu \mathrm{m}$ (Fig. 2b) also corresponding to the external diameter of the capillaries used as fluidics interconnections. If porous silicon is desired, a $500 \mathrm{~nm}$ thick LPCVD low-stress $\mathrm{Si}_{3} \mathrm{~N}_{4}$ thin film was first deposited (Fig. 2a) and was used as an etch mask during this step (Fig. 2c). Therefore, porous silicon was formed on all the unprotected area of the silicon micro-reactors, including the sidewalls of the micro-channels. In order to improve the adhesion of the carbon adsorbing powder that will be deposited afterwards in the micro-channels made or 
not of porous silicon, $25 \mathrm{~nm}$ of thermal oxide were grown to make them become hydrophilic and also to facilitate the bonding of carbon particles. Finally, the micro-channels were sealed with a Borofloat glass wafer by anodic bonding (Fig. 2d) and diced according to the dashed line showed in Fig. 2e revealing the inlet and outlet for the fluidic connectors.

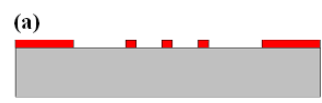

(c)

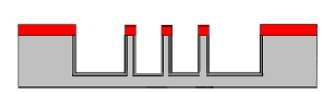

(e)

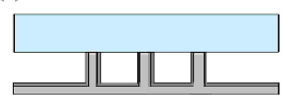

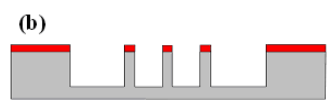

(d)

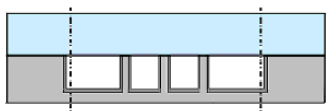

(f)

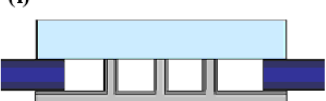

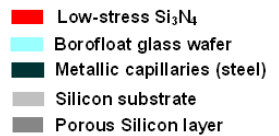

Figure 2: Fabrication process of the preconcentrator, which is illustrated here with the "Straight" design.

In order to have an autonomous component, for either the thermal treatments of the adsorbent materials and the desorption of the trapped gases, a platinum heater is integrated on the backside of the device by screen printing and the electrical connections are made with gold wires pasted on a platinum lacquer. This heating element is used to heat the device at temperatures up to $500{ }^{\circ} \mathrm{C}$ with a very homogeneous distribution of the temperature considering the good thermal conductivity of the silicon, as it was reported in our previous works $[1,2]$.

In order to have a good regulation of temperature, the $\mu$-preconcentrator is suspended in a metallic box (Fig. 3).

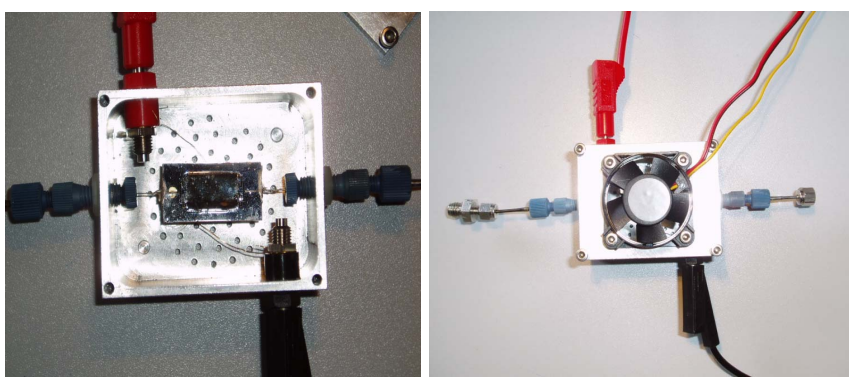

Figure 3: Micro-channel in porous silicon filled with carbon with metallic gas capillaries and suspended in metallic box.

\section{Adsorbent deposition}

The adsorbent insertion in micro-channels has been realized by two methods. The first one is a deposition using a fluidic solution [2], and the second one consists to deposit the adsorbent in a paste form before the sealing of the glass cover. The choice of the deposition method is closely related to the adsorbent nature.
These experiments in fluidic deposition have shown that the presence of pillars in the reaction chamber, particularly in "zigzag" design, is a key factor to homogenize the distribution of the liquid flow through the entire device and to enhance the carbon deposition. It was observed that the use of porous silicon has increased the quantity of deposited carbon in the micro-channels because of the presence of pores on the surface of the walls constitutes an important asset to get a good binding layer and thus to increase the quantity of adsorbent filled in the micro-channel compared to non-porous silicon.

Once the devices filled with adequate adsorbent material, the fluidic connections are made with metallic capillaries (steel) having $220 \mu \mathrm{m}$ of internal diameter and fixed with ceramic cement using a special procedure so as not to block the connectors during the sealing (Fig. 2f).

\section{Optimization of experimental parameters}

Five main parameters are of importance for the optimum operation of a preconcentrator device with a given geometry and filled with a fixed quantity of specific absorbent material, the gas flow rate during the gas adsorption, the gas flow rate during the desorption phases, the heating rate during the gas desorption phase, the duration of the gas adsorption and desorption phases, and the gas volume of the detector.

The miniaturization of such device imposes us a limitation on the gas flow rate, but for preconcentration, high flow rates are preferred in order to increase preconcentration factor for a given time of adsorption. The adsorption phase flow was set at $167 \mathrm{~mL} / \mathrm{min}$, which allowed us to inject a significant quantity of pollutant in a short time, while the phase desorption flow was maintained at $33 \mathrm{~mL} / \mathrm{min}$ to avoid an important dilution of the desorbed quantity. Likewise, to avoid a considerable detection volume, the metallic capillary at the outlet of the micro-preconcentrator was directly connected to the inside of the detector through its packaging (Sensor from FIGARO ref. 2620), and the latter was then calibrated in order to correct the influence of the gas flow on the characteristics of its response (Fig. 4).

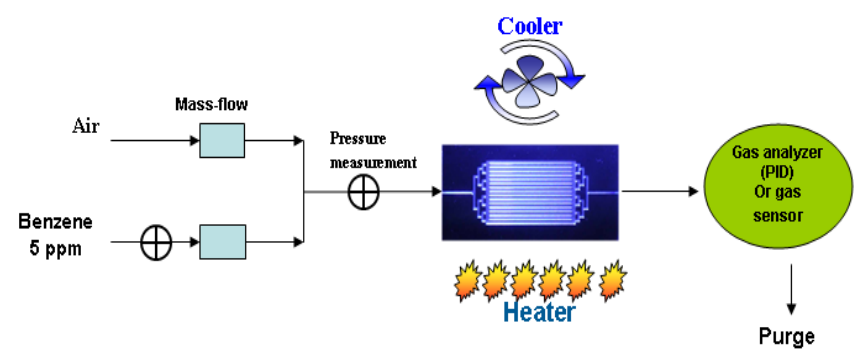

Figure 4: Schematic illustration of the micro-preconcentrator test bench.

In fact, it is well known, that the maximum of the desorption peak is an increasing function of the heating 
rate. Higher the heating rate is, narrower and higher the desorption peak is i.e. the maximum of the desorption more important and consequently a significant factor of preconcentration (Fig. 5). According to this observation, the preconcentration tests were performed with a heating rate of $160{ }^{\circ} \mathrm{C} / \mathrm{min}$, which corresponds to the maximum rate that can reach the heating resistance deposited on the backside of the micro-channels.

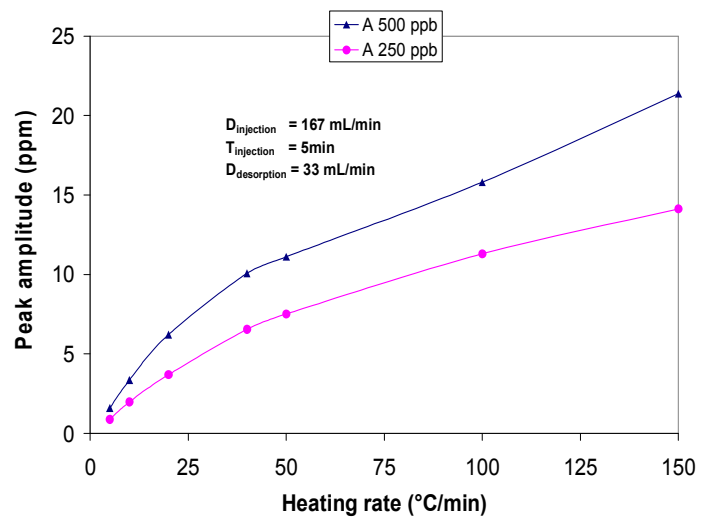

Figure 5: Demonstration of the dependence between the amplitude of the desorption peak and the heating rate.

Finally, the increase of the adsorption time can lead to an enhancement of the desorption peak amplitude, but in the same time causes a longer preconcentration cycle which for some applications might not be desirable. To this end, the adsorption and desorption times were maintained constant all through the experiences and lasted for 5 minutes since for these targeted applications as the pollution monitoring, a short preconcentration cycle is more appropriate.

So, it is very difficult to define a preconcentration factor since, for a given preconcentrator, the "amplification" of the concentration is closely related to these four external parameters. However, we will measure a "practical" preconcentration factor which we defined as the ratio between the concentration of the injected gas and the maximum of the measured concentration during the desorption phase.

\section{RESULTS AND DISCUSSIONS}

\section{Thermal Programmed Desorption (TPD) experiments}

The experiments in TPD have shown that carbon nanopowder adsorbs a significant quantity of water vapor thus reducing intensely its sensitivity to other gases in opposition to SWCNTs which have a low affinity with water (Fig. 6, 7).

In addition, the temperature of the maximum amplitude of the desorption peak varies with the adsorbent material. It is estimated at about 200 and $300{ }^{\circ} \mathrm{C}$ respectively for activated carbon nanopowder and SWCNTs. The increase of the desorption temperatures observed with the SWCNTs reflect a large activation energy of the desorption synonymous with a strong link between the adsorbent and adsorbed gas which is a bit harmful for the reduction of the power budget.

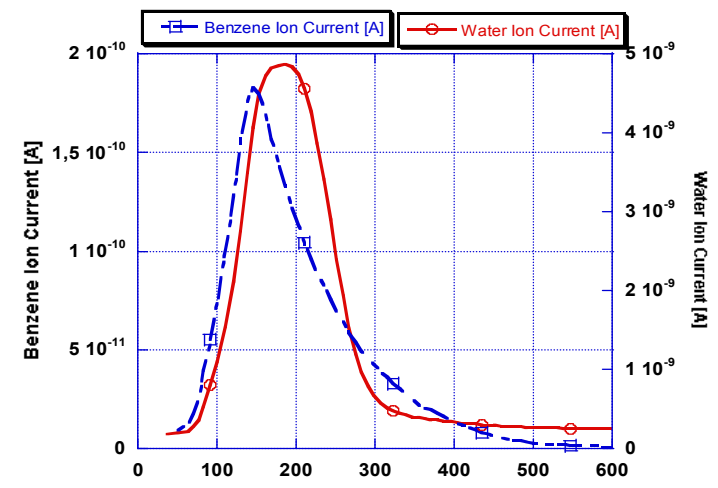

Figure 6: TPD spectrum of Carbon nanopowder after benzene adsorption.

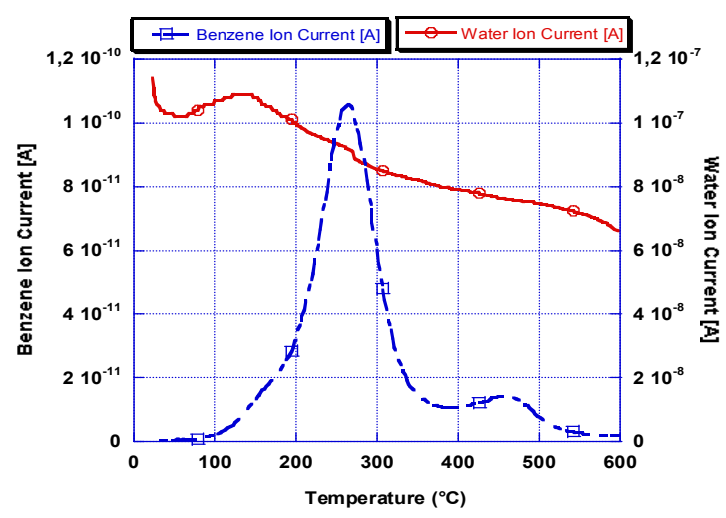

Figure 7: TPD spectrum of SWCNTs after benzene adsorption.

\section{Preconcentration}

Following the conclusions drawn from the previous experiments presented in the sections above, the preconcentration tests have been performed on silicon and porous silicon micro-channels with a "zigzag" design. In order to study in similar conditions the effect of optimized parameters, the quantity of deposited adsorbent on all micro-channels will be sensitive equal to about $1 \mathrm{mg}$.

On one hand, the tests of these porous silicon microchannels with carbon deposition have shown that the porous aspect of the micro-channels constitutes an important contribution to the performances of the preconcentrator. This result shows that the porous silicon, besides increasing the quantity of deposited carbon, contributes to the effectiveness of the gas preconcentration by making the desorption easier and thereby reducing the desorption temperature and at last the duration of the preconcentration cycle (Fig. 8).

This complete desorption of the couple porous siliconcarbon is, maybe, due to its activation energy of desorption 
lower than the pair non porous silicon-carbon one. The maximum of concentration obtained during desorption is about $14 \mathrm{ppm}$ corresponding to a "practical" factor of preconcentration (as defined above) of about 56.

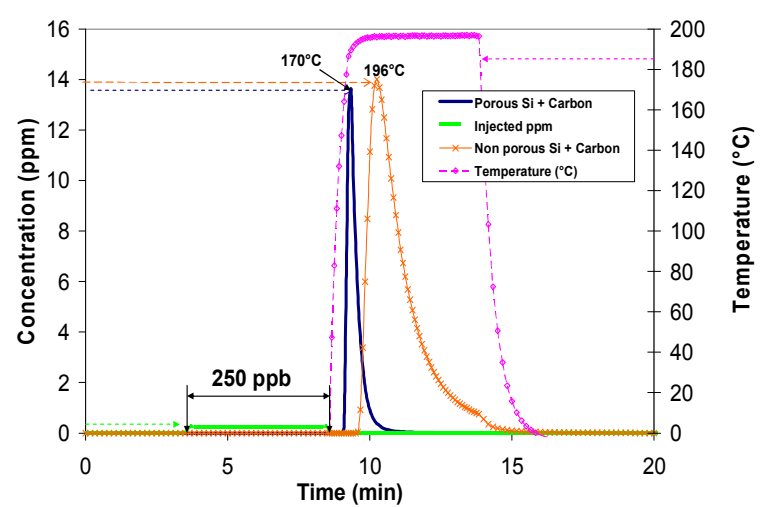

Figure 8: Comparison of gas sensor response between porous silicon and non porous silicon micro-channels filled with carbon nanopowder after an adsorption step of $250 \mathrm{ppb}$ and desorption step at $200^{\circ} \mathrm{C}$ with a heating rate of $160^{\circ} \mathrm{C} / \mathrm{min}$.

On other hand, the preconcentration tests of the non porous silicon micro-channels filled with SWCNTs have shown that the influence of water vapor in the performances of the micro-preconcentrator is lower than the one filled with activated carbon nanopowder with which the sensor response during the desorption is intensely reduced in presence humidity (Fig. 9, 10).

However, it should be noted that the desorption parameters of the non porous silicon micro-channels filled with SWCNTs are not optimum since we have observed a low factor of preconcentration and a spread of its desorption peak, which is synonym of a insufficient heating rate at $160^{\circ} \mathrm{C} / \mathrm{min}$. Some experiments are underway to optimize the preconcentration characteristics of the device when using SWCNTs.

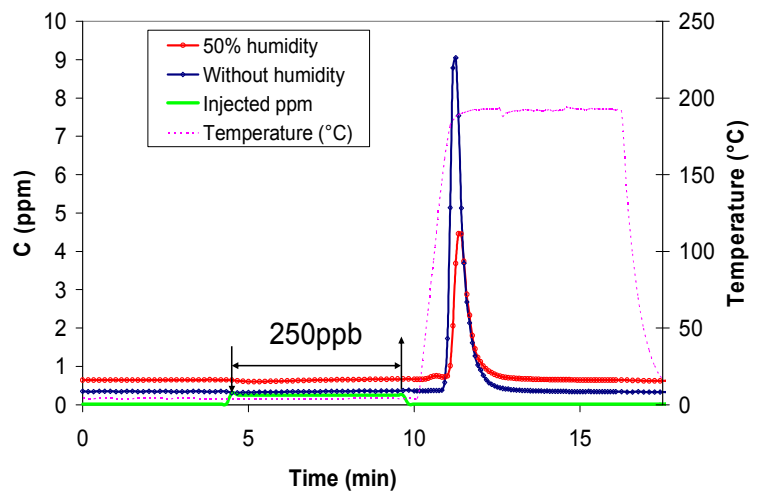

Figure 9: Photon Ionization Detector (PID) response of a silicon micro-channel filled with activated carbon nanopowder with and without humidity, when exposed to 5 min to $250 \mathrm{ppb}$ of benzene and desorbed during 5 min at a temperature of $200^{\circ} \mathrm{C}$.

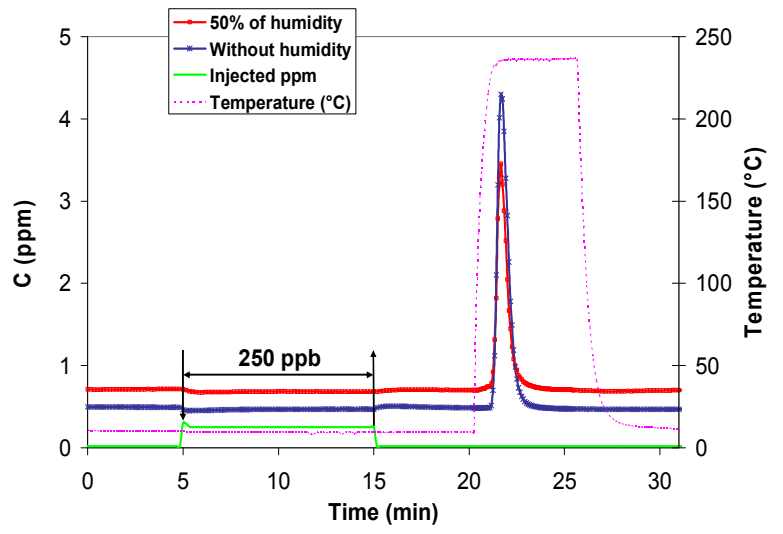

Figure 10: PID response of silicon micro-channel filled with SWCNTs with and without humidity when exposed to 5 min to $250 \mathrm{ppb}$ of benzene and desorbed at $240^{\circ} \mathrm{C}$.

\section{CONCLUSIONS}

The effect of gas preconcentration in micro-channels has been demonstrated and the use of two different adsorbent materials has been evaluated in a presence or not of relative humidity. To this end, SWCNT appears as adequate adsorbent for preconcentration of benzene because of its high specific area and adsorption capacity and its low affinity with humidity. Our new strategy is to focus on the functionalization of the SWCNTs in order to increase its affinity with benzene molecules.

Moreover, we are currently studying the preconcentration of other volatile compounds such as nitrobenzene which is being strongly adsorbed on activated carbon nanopowder and nanotubes making its desorption very difficult even with a temperature of about $400{ }^{\circ} \mathrm{C}$. In this case, other alternative materials are required. Tenax TA polymer seems the ideal candidate for the preconcentration of nitrobenzene since the latter is completely desorbed below $250{ }^{\circ} \mathrm{C}$.

\section{REFERENCES}

[1] E.H.M. Camara, C. Pijolat, J. Courbat, P. Breuil, D. Briand, N.F. de Rooij, "Microfluidic channels in porous silicon filled with a carbon adsorbent for gas preconcentration" Transducers (2007) pp 249-252.

[2] C. Pijolat, M. Camara, J. Courbat, J-P. Viricelle, D. Briand, N.F. de Rooij, "Application of carbon nanopowders for gas micro-preconcentrator" Sensors and Actuators B, 127 (2007) 179-185.

[3] F. Zheng, D. L. Baldwin, L. S. Fifield, N. C. Anheier, Jr., C. L. Aardahl, and Jay W. Grate, "Single-walled carbon nanotube paper as a sorbent for organic vapour preconcentration” Anal. Chem. 2006, 78, 2442-2446.

\section{CONTACT}

*P. Breuil, tel: +334-77-42-01-44, pbreuil@emse.fr 